OPEN ACCESS

Edited by:

Virgil Dalm,

Erasmus Medical Center, Netherlands

Reviewed by:

Irina Khamaganova,

Pirogov Russian National Research

Medical University, Russia

Sharon Henry,

University of Maryland Medical Center,

United States

Minxue Shen,

Central South University, China

*Correspondence:

Surasak Saokaew

surasak.sa@up.ac.th

Pochamana Phisalprapa

coco_a105@hotmail.com

Specialty section:

This article was submitted to

Dermatology,

a section of the journal

Frontiers in Medicine

Received: 03 June 2021 Accepted: 18 October 2021

Published: 19 November 2021

Citation:

Chaomuang N, Khamnuan P, Chuayunan N, Duangjai A, Saokaew S and Phisalprapa P (2021) Novel

Clinical Risk Scoring Model for

Predicting Amputation in Patients With

Necrotizing Fasciitis: The ANF Risk

Scoring System.

Front. Med. 8:719830.

doi: 10.3389/fmed.2021.719830

\section{Novel Clinical Risk Scoring Model for Predicting Amputation in Patients With Necrotizing Fasciitis: The ANF Risk Scoring System}

\author{
Natthaya Chaomuang 1,2,3, Patcharin Khamnuan ${ }^{4}$, Nipaporn Chuayunan ${ }^{4}$, \\ Acharaporn Duangjai ${ }^{5,6,7,8}$, Surasak Saokaew ${ }^{1,2,3,5,6,7 *}$ and Pochamana Phisalprapa ${ }^{9 *}$ \\ ${ }^{1}$ Unit of Excellence on Clinical Outcomes Research and IntegratioN (UNICORN), School of Pharmaceutical Sciences, \\ University of Phayao, Phayao, Thailand, ${ }^{2}$ Division of Pharmacy Practice, Department of Pharmaceutical Care, School of \\ Pharmaceutical Sciences, University of Phayao, Phayao, Thailand, ${ }^{3}$ Center of Health Outcomes Research and Therapeutic \\ Safety (Cohorts), School of Pharmaceutical Sciences, University of Phayao, Phayao, Thailand, ${ }^{4}$ Department of Nursing, \\ Phayao Hospital, Phayao, Thailand, ${ }^{5}$ Unit of Excellence on Herbal Medicine, School of Pharmaceutical Sciences, University \\ of Phayao, Phayao, Thailand, ${ }^{6}$ Biofunctional Molecule Exploratory Research Group, Biomedicine Research Advancement \\ Centre, School of Pharmacy, Monash University Malaysia, Bandar Sunway, Malaysia, ${ }^{7}$ Novel Bacteria and Drug Discovery \\ Research Group, Microbiome and Bioresource Research Strength, Jeffrey Cheah School of Medicine and Health Sciences, \\ Monash University Malaysia, Bandar Sunway, Malaysia, ${ }^{8}$ Department of Physiology, School of Medical Sciences, University \\ of Phayao, Phayao, Thailand, ${ }^{9}$ Division of Ambulatory Medicine, Department of Medicine, Faculty of Medicine Siriraj Hospital, \\ Mahidol University, Bangkok, Thailand
}

Background: Necrotizing fasciitis (NF) is a life-threatening infection of the skin and soft tissue that spreads quickly and requires immediate surgery and medical treatment. Amputation or radical debridement of necrotic tissue is generally always required. The risks and benefits of both the surgical options are weighed before deciding whether to amputate or debride. This study set forth to create an easy-to-use risk scoring system for predicting the risk scoring system for amputation in patients with NF (ANF).

Methods: This retrospective study included 1,506 patients diagnosed with surgically confirmed NF at three general hospitals in Thailand from January 2009 to December 2012. All diagnoses were made by surgeons who strictly observed the guidelines for skin and soft tissue infections produced by the Infectious Diseases Society of America. Patients were randomly allocated to either the derivation $(n=1,193)$ or validation ( $n$ $=313$ ) cohort. Clinical risk factors assessed at the time of recruitment were used to create the risk score, which was then developed using logistic regression. The regression coefficients were converted into item scores, and the total score was calculated.

Results: The following four clinical predictors were used to create the model: female gender, diabetes mellitus, wound appearance stage 3 (skin necrosis and gangrene), and creatinine $\geq 1.6 \mathrm{mg} / \mathrm{dL}$. Using the area under the receiver operating characteristic curve (AuROC), the ANF system showed moderate power (78.68\%) to predict amputation in patients with NF with excellent calibration (Hosmer-Lemeshow $\chi^{2}=2.59 ; p=0.8586$ ). The positive likelihood ratio of amputation in low-risk (score $\leq 4$ ) and high-risk (score $\geq 7$ ) patients was 2.17 (95\%Cl: 1.66-2.82) and 6.18 (95\%Cl: 4.08-9.36), respectively. The ANF system showed good performance (AuROC 76.82\%) when applied in the validation cohort. 


\begin{abstract}
Conclusion: The developed ANF risk scoring system, which includes four easy to obtain predictors, provides physicians with prediction indices for amputation in patients with NF. This model will assist clinicians with surgical decision-making in this time-sensitive clinical setting.
\end{abstract}

Keywords: clinical risk scoring model, amputation, patients, necrotizing fasciitis, ANF risk scoring system

\section{INTRODUCTION}

Necrotizing fasciitis (NF) is a dangerous infection that causes widespread inflammation and necrosis of the soft tissues, and that most often affects the fascia and subcutaneous layers (1). The recommended treatments for patients with NF are emergency surgical debridement and broad-spectrum antibiotic therapy (2). Delayed treatment may lead to significant soft tissue loss and/or limb loss, as well as an increased risk of mortality $(3,4)$. Patients can experience severe morbidity, such as amputation and organ failure, even after receiving intensive treatment $(3,5,6)$. To save the life of a patient, an amputation or radical debridement of necrotic tissue is frequently needed (7). The risks and benefits of each operation are weighed before deciding whether to amputate or dramatically debride (8). NF is rare with a global incidence of $0.3 / 100,000$ per year, and it has been shown that timely diagnosis and surgical management can enhance patient outcomes (9). The fatality and amputation rates associated with NF were estimated to be $15-29 \%(3,5)$ and $20.3-26 \%(6,10)$, respectively. The registered amputation and mortality rates associated with NF in Thailand are $8.7-15.4 \%$ and $5.9-22.1 \%$, respectively $(11,12)$.

The microbes, risk factors, and causes associated with NF have been established, and classification systems have been developed to identify and score patients at high risk for NF (13-17). In 2004, the Laboratory Risk Indicator for NF (LRINEC) score was created for diagnostic NF (18). Among the models that have been created to classify and/or score a patient's risk of NF (14-16), no study has developed a risk-prediction scoring system for amputation in NF.

Accordingly, the aim of this study was to use the epidemiology of NF disease in Thailand to create an easy-to-use risk scoring system for predicting the risk scoring system for amputation in patients with NF (ANF). That score could be used in a routine clinical practice, and it will help physicians and medical team to use it for making decisions on patient care. In addition, our ultimate goal is to help a patient avoid amputation by early prediction and managing the factors related to disease progression.

\section{MATERIALS AND METHODS}

\section{Study Design and Study Size}

This was a retrospective cohort study. A prognostic prediction analysis and clinical score development study was conducted in accordance with the transparent reporting of a multivariable prediction model for individual prognosis or diagnosis (TRIPOD) statement (19). Ten outcome events per predictor component were used to determine the sample size (the EPV method) (20). Based on other similar scoring systems that collectively draw upon 10 variables, a total of 100 amputations NF were needed.

\section{Setting and Study Population}

From January 2009 to December 2012, the medical records of patients with surgically-confirmed NF were obtained from three general hospitals in Northern Thailand. The hospitals were Chiangrai Prachanukroh Hospital (a 600-bed tertiarycare medical center), Kamphaeng Phet Hospital (a 330-bed public hospital), and Phayao Hospital (a 400-bed secondary-care medical center).

In total, 1,506 patients with NF were enrolled. They were divided into two groups using random sampling by computer generation (4:1), Stata command "insample." The derivation cohort $(n=1,193)$ is a sample to develop ANF scoring system, and a validation cohort $(n=313)$ is to confirm accuracy and appropriateness of this scoring system. In our study, an NF with amputation was found in 127 patients (8.4\%). This detail is shown in Table 1.

Necrotizing fasciitis was defined by the presence of extensive necrosis affecting at least the fascia and subcutaneous tissue. Patients diagnosed with NF according to the standard definition were eligible for inclusion. Diagnoses were made by surgeons who strictly observed the guidelines for skin and soft tissue infections produced by the Infectious Diseases Society of America (21).

The stages of wound appearance were defined as a clinical stage of NF. Cutaneous manifestations of NF present when the disease progresses through stages 1, 2, and 3 (early, intermediate, and late stages). Wound appearance stage 1 (early): the disease was clinically presenting with swelling and erythema; stage 2 (intermediate): belb formation is an important diagnostic clue of NF. When present, it signals the onset of critical skin ischemia; stage 3 (late): indicates the development of tissue necrosis and skin gangrene, which are severe signs of NF infection $(21,22)$.

Severe pain was defined based on the patient complaint by use of a pain-assessment tool (numerical rating scale 7-10) according to the pain management guideline $2017(23,24)$.

\section{Ethics Approval}

The protocol for this study was approved by the Ethics Committee of Chiangrai Prachanukroh Hospital and the Faculty of Medicine, Chiang Mai University, Chiang Mai, Thailand (032/2013; research ID: 1461; study code: COM-13-1461-EX).

\section{Statistical Analyses}

Descriptive statistics were used to summarize patient demographic and clinical characteristics. Continuous data 
TABLE 1 | Clinical and demographic characteristics of patients with necrotizing fasciitis among all patients, and compared between the derivation cohort and the validation cohort.

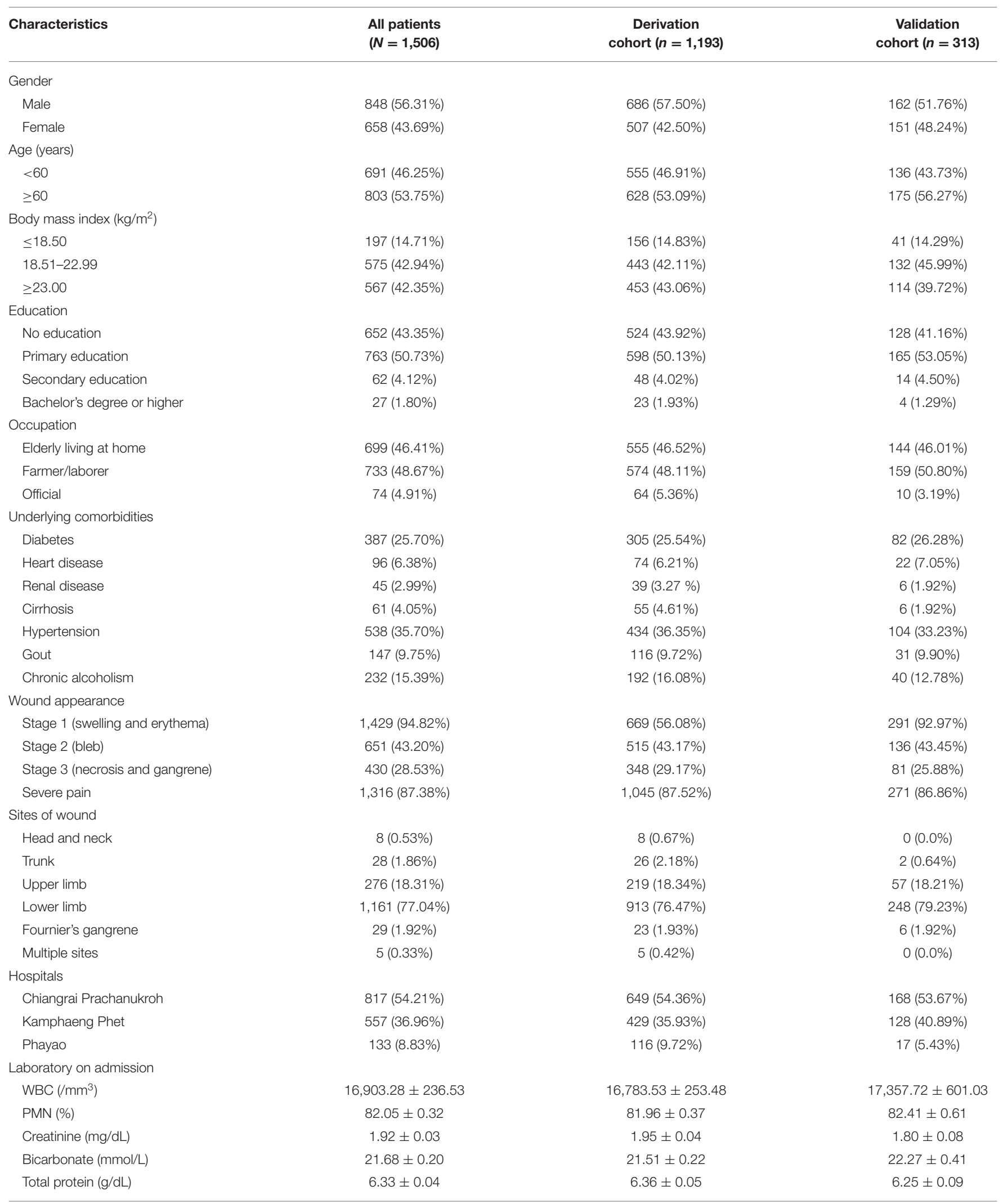


TABLE 1 | Continued

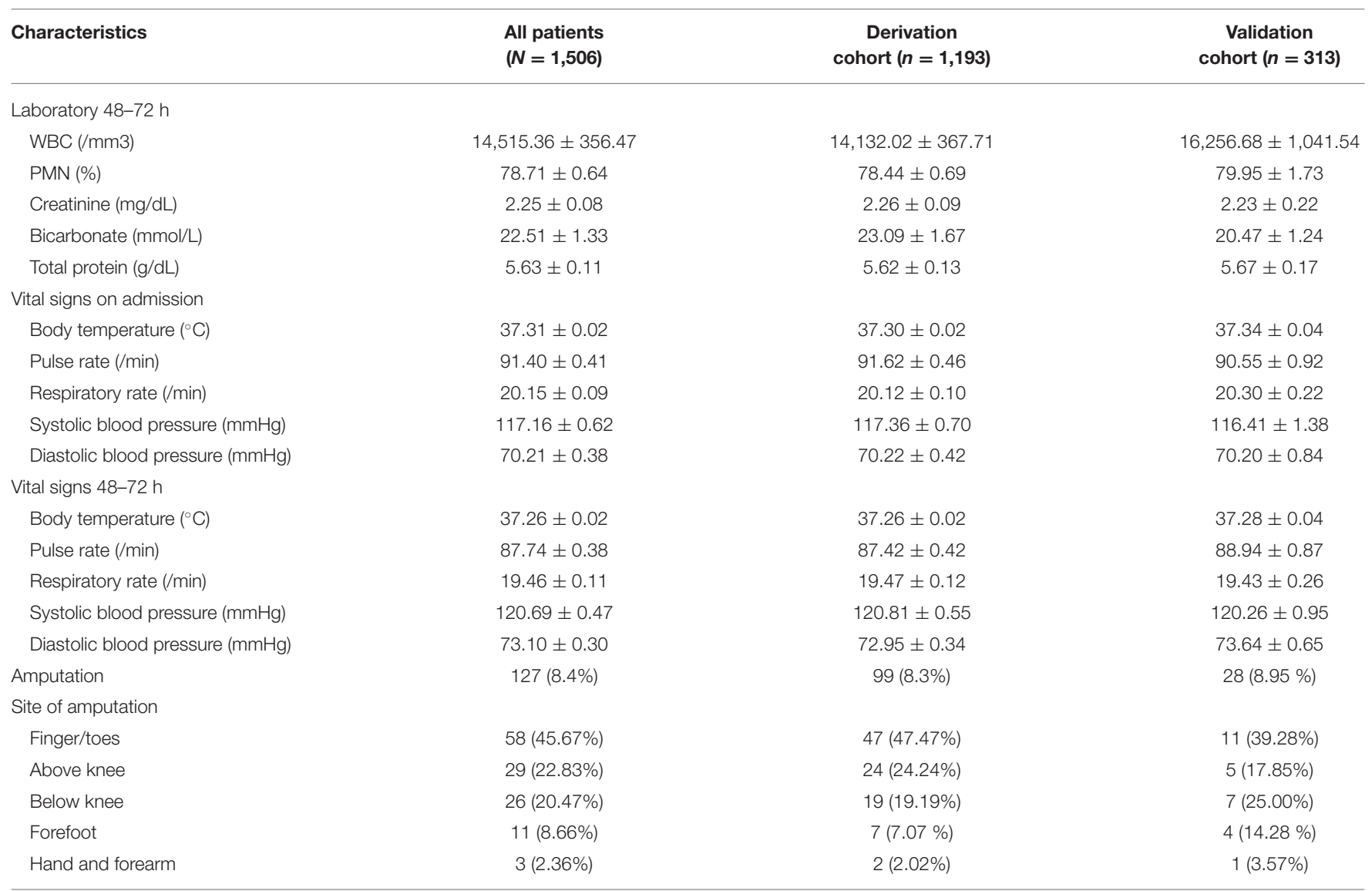

Data presented as mean \pm standard deviation or number and percentage.

WBC, white blood cell count; PMN, polymorphonuclear cell or neutrophil.

were compared using Student's $t$-test, and those results are shown as mean \pm standard deviation (SD). Categorical data were compared using chi-square test or Fisher's exact test, and those results are given as number and percentage. All $p$-values are two-tailed and were considered statistically significant at a $p$-value $<0.05$.

\section{Model Development}

Variables that could affect the outcomes were established and incorporated into the development model. The predictive variables were selected by factors reported from previous studies to ensure a clinical significance (25-27). Clinically meaningful and interpretable cut-off points were used to transform continuous variables into categorical variables. In addition, we also selected the parameters from statistical analysis that $P$-values $<0.05$ were considered statistically significant to include in running the model. Amputation and no amputation variables were evaluated using univariable logistic regression, and those results are summarized as odds ratio (OR) and 95\% confidence interval (CI). Variables found to be statistically significant and other variables of interest were then entered into a multivariable logistic regression model to identify factors independently associated with amputation in NF. Item scores were calculated using the weighted coefficients of the significant variables from multivariable analysis. By dividing each regression coefficient by the smallest coefficient of the model and rounding to the nearest integer, the score was determined (28-30). To determine the power of the model to discriminate between amputation and no amputation, a receiver operating characteristic (ROC) curve was plotted, and the area under the ROC curve (AuROC) was also calculated. A Hosmer-Lemeshow goodness of fit test was also performed (31). Cut-off scores were determined to classify the patients with NF as low-risk, moderate-risk, or high risk for death. Sensitivity, specificity, positive predictive value (PPV), negative predictive value (NVP), positive likelihood ratio ( $\mathrm{LR}+$ ), and negative likelihood ratio (LR-) were calculated to evaluate the diagnostic performance of the model (32).

\section{Model Validation}

The validation cohort $(n=313)$ was used to verify the scoring system. The efficiency and accuracy of the model were assessed using a ROC curve. The same diagnostic performance parameters that were calculated and assessed in the derivation cohort were calculated and assessed in the validation cohort. 


\section{RESULTS}

\section{Patient Characteristics}

A total of 1,506 patients with NF were enrolled. Their ages ranged from 2 to 95 years, $43.69 \%$ were female, $85.29 \%$ had a body mass index (BMI) $\geq 18.50$, and $25.70 \%$ had diabetes mellitus. A swelling wound (wound appearance stage 1) was presented in $94.82 \%$ of patients, and the most common wound site was the lower limbs (77.04\%). An amputation with NF found 127 patients $(8.4 \%)$ and non-amputation with NF found 1,379 patients $(91.6 \%)$. Sites of amputation were finger/toes $(44.1 \%)$, above knee (22.8\%), below knee (20.5\%), forefoot (8.7\%), hand and forearm (3.9\%). Clinical and demographic characteristics of patients with NF among all patients and comparison between the derivation cohort and the validation cohort are shown in Table 1.

\section{Predictors of Amputation Patients With NF}

The overall amputation rate in this study was 8.43\% (127 of 1,506 patients). Univariable analysis for risk factors significantly associated with amputation in patients with NF in the derivation cohort is shown in Table 2. Factors found to be significantly associated with amputation were female gender, age, education, heart disease, hypertension, erythematous wound, bleb wound, white blood cell count (WBC), percentage of polymorphonuclear cell or neutrophil, serum creatinine, serum bicarbonate, pulse rate, systolic blood pressure, diastolic blood pressure, severe sepsis, and length of hospital stay.

\section{Model Development}

The variables identified in univariable analysis were entered into subsequent multivariable analysis to develop the scoring system for amputation in patients with NF. Using backward stepwise logistic regression, the multivariable model included four variables. The weighted coefficient was used to calculate the point value for each factor, which was then rounded to the nearest whole number (Table 3). The total prediction score comprises the summation of the following four scores: female gender (yes $=1$, no $=0$ ); diabetes mellitus (yes $=3$, no $=0$ ); wound appearance stage 3 (yes $=3$, no $=0$ ); and creatinine $\geq 1.6 \mathrm{mg} / \mathrm{dL}$ (yes $=2$, no $=0$ ). The highest possible score is 9 , and a higher score indicates a higher risk for amputation.

To categorize patients into three risk categories, cut-off scores of 4 and 7 were selected. Patients with a total score of $\geq 7$ were considered to be at high risk. Amputation among those patients was found to be predicted with moderate accuracy (28/78 cases; PPV 35.89\%). Patients with a total score of $\leq 4$ were considered to be at low risk. No amputation was correctly predicted in $95.99 \%$ of cases (863/899) in this group. No amputation group could be excluded with moderate accuracy (NPV 21.43\%). The corrected prediction of absence or presence of amputation was $(863+$ $28) /(899+78)=91.19 \%$, whereas the incorrect prediction rate was $(36+50) /(899+78)=8.80 \%$ (Table 4$)$. With this scoring system and using two cut-off points, the risk score discriminated between patients with NF with a risk for amputation and those without a risk for amputation with high validity (AuROC $76.68 \%$ ) (Figure 1). The predictive model was also shown to be well-calibrated (Hosmer-Lemeshow $\chi^{2}=2.59 ; p=0.8586$ ).
TABLE 2 | Univariable analysis for risk factors significantly associated with amputation in patients with necrotizing fasciitis in the derivation cohort.

\begin{tabular}{|c|c|c|c|}
\hline \multirow[t]{2}{*}{ Variables } & \multicolumn{3}{|c|}{ Derivation cohort $(n=1,193)$} \\
\hline & Odds ratio & $\begin{array}{l}95 \% \mathrm{Cl} \text { of } \\
\text { odds ratio }\end{array}$ & $p$-value \\
\hline Female gender & 1.62 & $1.05-2.51$ & 0.0203 \\
\hline Age (per year) group & 1.37 & $0.88-2.15$ & 0.1381 \\
\hline Body mass index & 1.00 & $0.63-1.60$ & 0.9897 \\
\hline Education & 1.33 & $0.86-2.07$ & 0.1670 \\
\hline Occupation & 1.34 & $0.87-2.09$ & 0.1610 \\
\hline \multicolumn{4}{|l|}{ Underlying comorbidities } \\
\hline Diabetes & 4.24 & $2.72-6.61$ & $<0.0001$ \\
\hline Heart disease & 1.80 & $0.79-3.69$ & 0.0942 \\
\hline Renal disease & 1.65 & $0.49-4.39$ & 0.2988 \\
\hline Cirrhosis & 0.19 & $0.01-1.17$ & 0.0745 \\
\hline Hypertension & 1.32 & $0.85-2.05$ & 0.1854 \\
\hline Gout & 0.80 & $0.32-1.71$ & 0.5646 \\
\hline Chronic alcoholism & 1.77 & $0.89-3.90$ & 0.0902 \\
\hline \multicolumn{4}{|l|}{ Wound appearance } \\
\hline Stage 1 (swelling and erythema) & 1.41 & $0.90-2.22$ & 0.1135 \\
\hline Stage 2 (bleb) & 0.80 & $0.51-1.25$ & 0.3155 \\
\hline Stage 3 (necrosis and gangrene) & 4.31 & $2.75-6.76$ & $<0.0001$ \\
\hline Severe pain & 0.78 & $0.43-1.50$ & 0.4028 \\
\hline \multicolumn{4}{|l|}{ Sites of wound } \\
\hline Upper limb & 0.71 & $0.36-1.29$ & 0.2579 \\
\hline Lower limb & 1.41 & $0.82-2.55$ & 0.1948 \\
\hline Multiple sites & 2.38 & $0.57-7.36$ & 0.1104 \\
\hline \multicolumn{4}{|l|}{ Laboratory on admission } \\
\hline WBC $\left(/ \mathrm{mm}^{3}\right)$ & 1.63 & $0.75-3.23$ & 0.1457 \\
\hline PMN (\%) & 0.93 & $0.60-1.45$ & 0.7665 \\
\hline Creatinine (mg/dL) & 2.34 & $1.49-3.73$ & 0.0001 \\
\hline Bicarbonate (mmol/L) & 1.47 & $0.35-4.49$ & 0.4820 \\
\hline Total protein (g/dL) & 1.08 & $0.02-8.01$ & 0.9375 \\
\hline \multicolumn{4}{|l|}{ Laboratory 48-72 h } \\
\hline WBC $\left(/ \mathrm{mm}^{3}\right)$ & 0.79 & $0.15-2.73$ & 0.7148 \\
\hline PMN (\%) & 1.21 & $0.60-2.36$ & 0.5529 \\
\hline Creatinine (mg/dL) & 1.35 & $0.74-2.49$ & 0.2826 \\
\hline Bicarbonate (mmol/L) & 0.00 & $0.00-3.03$ & 0.2453 \\
\hline Total protein (g/dL) & 0.00 & $0.00-4.27$ & 0.3240 \\
\hline \multicolumn{4}{|l|}{ Vital signs on admission } \\
\hline Body temperature $\left({ }^{\circ} \mathrm{C}\right)$ & 1.34 & $0.84-2.10$ & 0.1769 \\
\hline Pulse rate (/min) & 1.10 & $0.12-4.64$ & 0.8977 \\
\hline Respiratory rate (/min) & 2.30 & $0.36-95.4$ & 0.4026 \\
\hline Systolic blood pressure (mmHg) & 0.92 & $0.39-1.90$ & 0.8294 \\
\hline Diastolic blood pressure (mmHg) & 1.08 & $0.41-2.45$ & 0.8378 \\
\hline \multicolumn{4}{|l|}{ Vital signs 48-72 h } \\
\hline Body temperature $\left({ }^{\circ} \mathrm{C}\right)$ & 1.62 & $0.95-2.67$ & 0.0488 \\
\hline Pulse rate (/min) & 0.00 & $0.00-3.61$ & 0.3007 \\
\hline Respiratory rate (/min) & 0.91 & $1.42-1.77$ & 0.7807 \\
\hline Systolic blood pressure (mmHg) & 1.28 & $0.14-5.61$ & 0.7370 \\
\hline Diastolic blood pressure (mmHg) & 5.42 & $1.41-17.79$ & 0.0007 \\
\hline
\end{tabular}

A p-value $<0.05$ indicates statistical significance.

WBC, white blood cell count; PMN, polymorphonuclear cell or neutrophil. The bold and italic values mean $p$ value $<0.05$ indicates statistic significant. 
TABLE 3 | Multivariable analysis and risk score for amputation in patients with necrotizing fasciitis.

\begin{tabular}{|c|c|c|c|c|c|}
\hline Predictors & Coefficient & aOR & $95 \% \mathrm{Cl}$ of aOR & $p$ & Assigned score \\
\hline Female gender & 0.4593086 & 1.58 & $1.01-2.47$ & 0.043 & 1 \\
\hline Diabetes mellitus & 1.2440160 & 3.47 & $2.23-5.41$ & $<0.001$ & 3 \\
\hline Wound appearance: stage 3 & 1.4157790 & 4.11 & $2.71-6.25$ & $<0.001$ & 3 \\
\hline Creatinine $\geq 1.6 \mathrm{mg} / \mathrm{dL}$ & 0.9409972 & 2.56 & $1.62-4.05$ & $<0.001$ & 2 \\
\hline
\end{tabular}

$p$-value $<0.05$ indicates statistical significance.

aOR, adjusted odds ratio; $\mathrm{Cl}$, confidence interval.

The bold and italic values mean $p$ value $<0.05$ indicates statistic significant.

TABLE 4 | Distribution of risk of amputation in patients with necrotizing fasciitis, diagnostic performance, and interpretation in the derivation cohort ( $n=1,193$ ).

\begin{tabular}{lccc}
\hline Derivation cohort & Low risk (score $\mathbf{4}$ ) & Moderate risk (score 5-6) & High risk (score $\geq$ 7) \\
\hline Total & 899 & 216 & 78 \\
No amputation & 863 & 181 & 50 \\
Amputation & 36 & 35 & 28 \\
Diagnostic performance & & & 1,193 \\
Sensitivity & $78.88 \%$ & 994 \\
Specificity & $63.63 \%$ & & $28.28 \%$ \\
Positive predictive value & $95.99 \%$ & $95.43 \%$ \\
Negative predictive value & $21.43 \%$ & $35.89 \%$ \\
Likelihood ratio $(+)$ & $2.17(95 \% \mathrm{Cl}: 1.66-2.82)$ & $93.63 \%$ \\
Likelihood ratio $(-)$ & $0.33(95 \% \mathrm{Cl}: 0.27-0.40)$ & $6.18(95 \% \mathrm{Cl}: 4.08-9.36)$ \\
\hline
\end{tabular}

Cl, confidence interval.

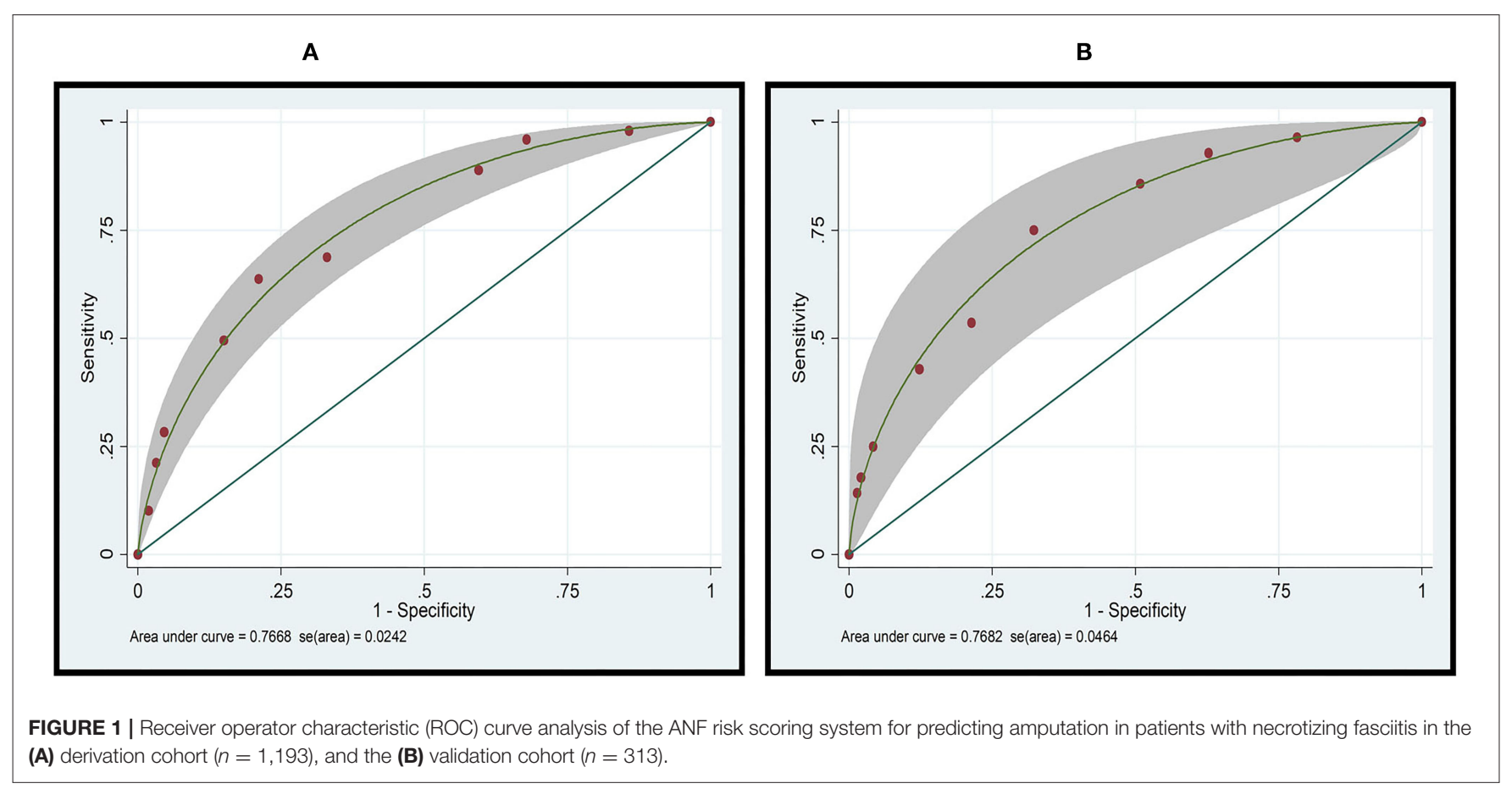

\section{Model Validation}

The ROC curves for the derivation and validation cohorts showed similar results (AuROC 76.68 and $76.82 \%$, respectively; Figure 1). Amputation in the high-risk group was accurately predicted in $36.84 \%$ of cases, and the presence of amputation was predicted with high moderate accuracy (PPV 36.84\%). In the lowrisk group, no amputation was predicted in 94.51\% (224/237) of the cases, with the absence of amputation being predicted 
TABLE 5 | Distribution of risk of amputation in patients with necrotizing fasciitis, diagnostic performance, and interpretation in the validation cohort ( $n=313$ ).

\begin{tabular}{|c|c|c|c|c|}
\hline Validation cohort & Low risk (score $\leq 4$ ) & Moderate risk (score 5-6) & High risk (score $\geq 7$ ) & Total \\
\hline Total & 237 & 57 & 19 & 313 \\
\hline No amputation & 224 & 49 & 12 & 285 \\
\hline Amputation & 13 & 8 & 7 & 28 \\
\hline \multicolumn{5}{|l|}{ Diagnostic performance } \\
\hline Sensitivity & $78.59 \%$ & & $25.00 \%$ & \\
\hline Specificity & $53.57 \%$ & & $95.78 \%$ & \\
\hline Positive predictive value & $94.51 \%$ & & $36.84 \%$ & \\
\hline Negative predictive value & $19.74 \%$ & & $92.86 \%$ & \\
\hline Likelihood ratio (+) & 1.69 (95\%Cl: 1.13-2.53) & & 5.94 (95\%Cl: 2.54-13.85) & \\
\hline Likelihood ratio (-) & 0.39 (95\%Cl: 0.27-0.00) & & 0.78 (95\%Cl: 0.63-0.97) & \\
\hline
\end{tabular}

Cl, confidence interval.

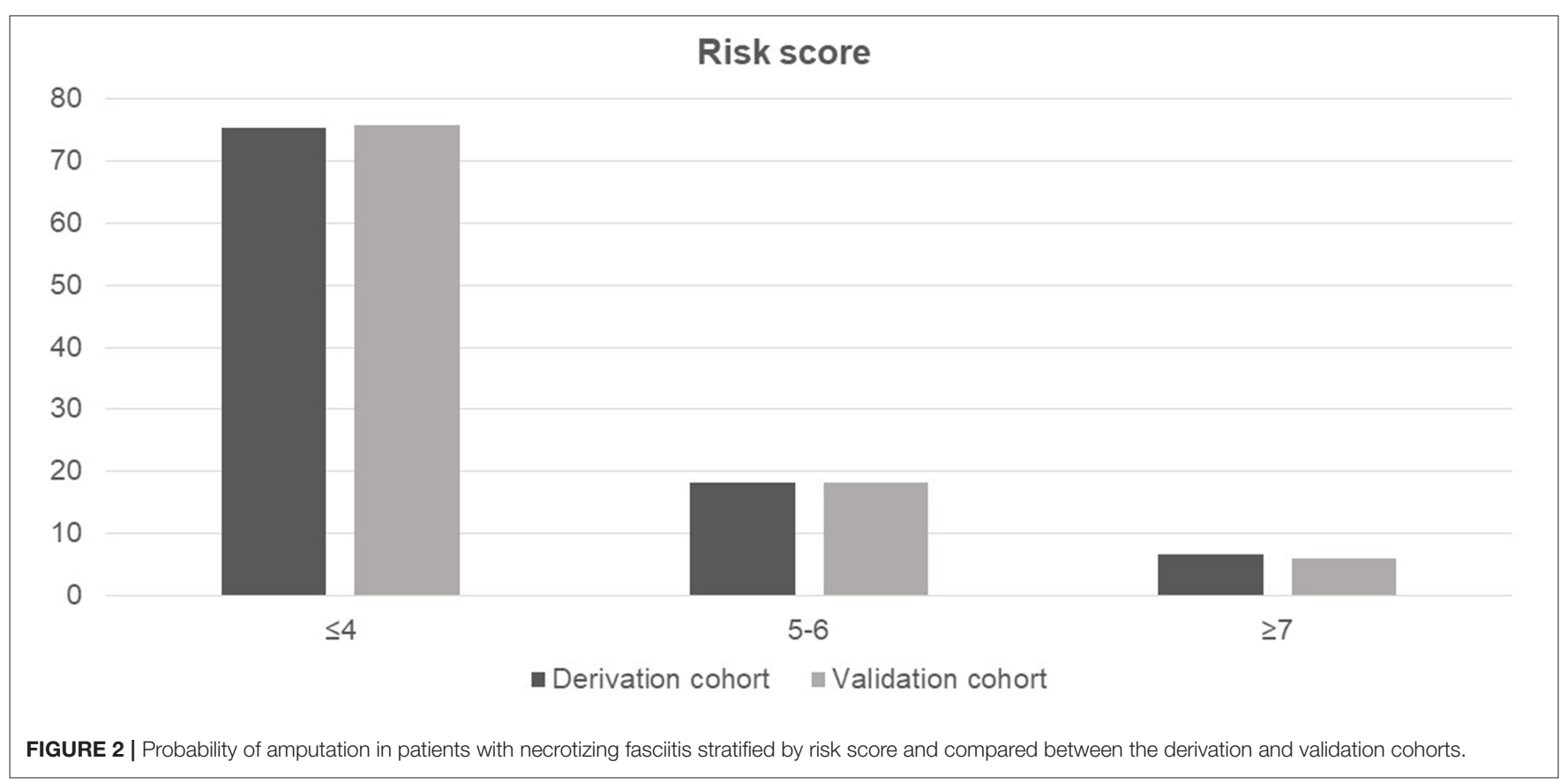

with moderate accuracy (NPV 34.75\%). The accurate prediction of amputation or no amputation was $(224+7) /(237+19)=$ $90.23 \%$, whereas the incorrect prediction rate was $(13+12) /(237$ $+19)=9.76 \%$ (Table 5).

The probability of amputation in patients with NF stratified by risk score and compared between the derivation and validation cohorts is shown in Figure 2. Patients were classified into three groups based on the calculated cut-off values, as follows: low-risk (score $\leq 4$ ), moderate-risk (score of 5-6), or high-risk (score $\geq 7$ ). All comparisons among the three risk groups were statistically significant with $p$-values $<0.001$ for all comparisons in both the cohorts.

\section{DISCUSSION}

In this study, we developed a clinical risk scoring system to predict amputation in patients with NF (the ANF risk scoring system) that can be used as a screening tool to identify NF patients at risk for amputation. The ANF risk scoring system grades NF patients as being at low risk, moderate risk, or high risk for amputation, and this information will help clinicians with treatment decision-making. Amputation is an intensive treatment that can cause significant morbidity and organ failure; however, patients with high mortality risk are often indicated for primary amputation to prevent death. The ANF tool may be useful in decision-making for the clinical management of patients with NF who have been admitted to the hospital.

We divided the patients in the NF patients in the derivation group into the amputation and no amputation groups. We then analyzed for factors that were significantly different between those two groups. Significant factors from univariable analysis were then entered into a multivariable logistic regression model to identify predictors independently associated with amputation. The ANF risk scoring system includes predictors related to a patient demography (female gender), patient comorbidity (diabetes mellitus), clinical sign (wound appearance stage 3, 
presenting with gangrene and necrosis of the skin), and laboratory result (creatinine $\geq 1.6 \mathrm{mg} / \mathrm{dL}$ ). These predictors are similar to the important risk factors for amputation identified by many other studies (27).

The patients with NF in this study were classified into three groups according to their likelihood of amputation (low risk, moderate risk, or high risk). The ANF risk scoring system was internally validated, and it showed high discriminative power when evaluated in this study's validation cohort. The cut-off points used in this study were determined from evaluations of sensitivity, specificity, and positive and negative predictive values. The cut-off point for high risk for amputation was determined to be an ANF score of $\geq 7$. Using this system, patients scoring $\leq 4$ are classified as low-risk, and patients scoring 5-6 are classified as being at moderate risk for amputation. The ANF score of a patient can then be combined with other factors and the judgment of the physician. Patients with an ANF score $\geq 7$ should be closely monitored and managed to avoid limb loss or death, if possible.

The ANF score developed in this study differs from the LRINEC score $(14,15,18)$. The tool is based on six laboratory variables at the time of presentation, including C-reactive protein (CRP), total white cell count, hemoglobin, serum sodium, creatinine, and glucose. The LRINEC score is a reliable tool that can aid in the clinical diagnosis of NF (18). It can also identify high-risk patients and predict NF outcome (33). In 2008, Su et al. reported that patients with a LRINEC score of $\geq 6$ had a significantly higher rate of amputation $(P=0.002)(16)$. In contrast, Leiblien et al. reported that a high LRINEC score $(\geq 8$; $p=0.19)$ had no significant association with the amputation rate (34). The LRINEC score examines six laboratory-based parameters, but no clinical parameters, such as comorbidities and clinical observations, are taken into consideration (14). In contrast to the LRINEC score, the ANF risk scoring system was developed specifically to predict amputation in patients with NF. We utilize the LRINEC score to predict amputation prognosis in our data. We found the LRINEC score had low sensitivity (51.94 vs. $78.88 \%$ ) and specificity (92.33 vs. $95.43 \%$ ) compared with our score (the ANF risk scoring system) in our study.

Women have more subcutaneous fat than men, which makes them more vulnerable to infection. Previous studies have shown that female gender, diabetes, cutaneous gangrene on admission, symptoms of an underlying condition, clostridial infection, heart disease, and shock (systolic blood pressure $<90 \mathrm{mmHg}$ ) at hospital admission are all independent predictors of limb loss in patients with NF $(5,35)$.

Diabetes was previously reported to be a clinical predictor of limb amputation in patients with NF. In our study, the prevalence of diabetes was $25.70 \%$. Our finding that diabetes is independently associated with limb loss is similar to the reported findings of several previous studies $(5,34,36,37)$. Hyperglycemia causes bacterial growth and tissue ischemia as a consequence of peripheral vascular disease. Diabetes is, therefore, associated with worse outcomes in patients with NF, including amputation. Atherosclerosis is also common in diabetic patients. Smallcaliber arteries were found to have more severe atherosclerotic changes in limb vessels, resulting in ischemia and gangrene, and eventually amputation $(36,37)$.

Wang et al. developed a classification of wound appearance stages based on clinical symptoms that facilitates rapid diagnosis and treatment (22). In the early stages of infection, this may include tenderness to palpation that extends beyond the visible region of skin involvement. Capillary leakage and elevated temperature cause erythema and swelling/edema. Blister and bullae formation are essential symptoms of skin ischemia in intermediate stage 2. Crepitus, skin anesthesia, and necrosis with a dusky discoloration occur in stage 3 (22). Wound appearance stage 3 with skin necrosis and gangrene was found to be a predictor of amputation in our research. Khamnuan et al. reported NF presenting with gangrene and skin necrosis at the time of diagnosis to be a strong predictor of amputation ( $p$ $<0.001$ ) (27). Similarly, previous studies reported cutaneous gangrene presented on admission to be significantly associated with a higher risk of amputation $(p=0.005)(4,6,7,38)$. Skin necrosis and gangrene were the most common causes of poor prognosis in late-stage NF cases (22). In most cases with NF, soft tissue swelling in the affected area was a common clinical symptom. Tissue edema and muscle necrosis are caused by infection that has spread across the lymphatic and vascular systems. Skin necrosis is caused by thrombosis of microvascular vessels and nerve supply dysfunction. A loss of blood flow causes gangrene, which is necrosis of the tissue. Depending on the extent of the necrotic areas, debridement or amputation was required whenever gangrene was present $(26,39)$.

A higher risk of limb failure was found in our sample when the serum creatinine level was $>1.6 \mathrm{mg} / \mathrm{dL}$. Renal dysfunction, which is thought to be linked with septic shock, is reflected by a higher serum creatinine level. Several previous studies reported shock (systolic blood pressure $<90 \mathrm{mmHg}$ ) at admission to be an independent predictor of limb loss, elevated creatinine level, and higher mortality (40).

\section{Strengths}

The strengths of this study should be emphasized. First, this study included a large sample size of patients with NF to evaluate amputation outcome. We also included patients from three large hospitals in Thailand, which suggests that our results can be generalized to other regions of Thailand, and to other low to middle income Asian countries. Second, the ANF risk scoring system was developed in accordance with the stringent criteria set forth in the TRIPOD statement (19). Third, the developed scoring system comprises only four variables, and all of them are easy to obtain and quantify. Interestingly, the predictors that were identified are different types of parameters. ANF scoring includes patient demographic data (being female), patient comorbidity data (diabetes mellitus), patient clinical characteristic data (wound appearance stage 3, presenting with skin necrosis and gangrene), and patient routine laboratory data (serum creatinine). Fourth, the ANF risk scoring system was validated using different patient data sets. In both the derivation and validation cohorts, the ANF score demonstrated good prediction ability and satisfactory diagnostic results. Fifth and last, since serum creatinine is the only laboratory test 
that is needed, this model is very affordable. The cost of this investigation is only 60 Thai baht (THB) (2 USD), and serum creatinine is a routine laboratory test that is routinely used to track patient health status while they are in the hospital.

\section{Limitations}

The most notable limitation of this study is it retrospective design, which rendered it vulnerable to missing or incomplete data. For example, the LRINEC scoring system could not be used in this study because some important laboratory investigations, such as CRP level, were not performed in all patients.

The data on the amputations index-date, time, and duration of index-operations were not collected because of the retrospective analysis from January 2009 to December 2012. However, since the reason of performing an amputation is to control the infection indicated by several outcomes, e.g., reducing lengthof-stay (LOS) or mortality rate, we found that LOS in a hospital of non-amputated is significantly lower ( 11 days, $\mathrm{SD}=11.59$ ) than those in amputated groups (16 days, $\mathrm{SD}=10.79), p<$ 0.001 . However, there was no difference in mortality between amputations and no amputations (20.20 vs. $19.12 \%$, Odds ratio 1.07: $95 \% \mathrm{Cl} 0.61-1.81)$. The data on the amputations index date, time, and duration of index operations were not collected. However, we have the data of length of stay in a hospital of non-amputated, which was 11 days $(\mathrm{SD}=11.59)$ and in amputated it was 16 days $(\mathrm{SD}=10.79)$, with a $P$-value $<0.001$. Amputation was performed to control the infection. However, there was no difference in mortality between amputations and no amputations. Our data showed that the mortality of amputated was $20.20 \%$ and non-amputated was $19.12 \%$ (Odds ratio 1.07; $95 \% \mathrm{Cl} 0.61-1.81)$. This was similar to previous studies $(1,26)$ which reported that amputation did not show the reduction of mortality, but patients who underwent amputation had to undergo fewer operations to control the infection and to achieve wound coverage. Kaplan-Meier survivorship analysis revealed that the survival rate decreased with delayed surgery and prolonged symptoms. Survival sharply declined with a delay in surgery of more than $24 \mathrm{~h}$.

Despite this limitation, the ANF risk scoring system can be used in all clinical settings because of its low-cost and because it is simple to implement and use. The advantage of the ANF score is that it can help to detect early the amputation risk in patients with NF. Using this system, disease progression can be stopped or reduced, potential complications of NF can be monitored, and the risk of amputation can be greatly reduced or eliminated.

\section{Conclusion}

The developed ANF risk scoring system, which includes four easy to obtain predictors, provides physicians with prediction

\section{REFERENCES}

1. Wong C-H, Chang H-C, Pasupathy S, Khin L-W, Tan J-L, Low C-O. Necrotizing fasciitis: clinical presentation, microbiology, and determinants of mortality. J Bone Joint Surg. (2003) 85:1454-60. doi: 10.2106/00004623-200308000-00005

2. Headley AJ. Necrotizing soft tissue infections: a primary care review. Am Fam Physician. (2003) 68:323-8. indices for amputation in patients with NF. This model will assist clinicians with surgical decision-making in this time-sensitive clinical setting.

\section{DATA AVAILABILITY STATEMENT}

The original contributions presented in the study are included in the article/Supplementary Materials, further inquiries can be directed to the corresponding authors at:coco_a105@hotmail.com.

\section{ETHICS STATEMENT}

The studies involving human participants were reviewed and approved by the Ethics Committee of Chiangrai Prachanukroh Hospital and the Faculty of Medicine, Chiang Mai University, Chiang Mai, Thailand (032/2013; research ID: 1461; study code: COM-13-1461-EX). Written informed consent for participation was not required for this study in accordance with the national legislation and the institutional requirements.

\section{AUTHOR CONTRIBUTIONS}

NCha, PK, and SS: study concept and design, statistical analysis, and interpretation of data. PK, NChu, and SS: acquisition of data. NCha, SS, and PP: drafting of the manuscript. NCha, PK, NChu, $\mathrm{AD}, \mathrm{SS}$, and PP: critical revision of the manuscript. All authors contributed to the article and approved the submitted version.

\section{FUNDING}

This study was supported by a grant from the University of Phayao via the Unit of Excellence on Clinical Outcomes Research and IntegratioN (UNICORN).

\section{ACKNOWLEDGMENTS}

The authors thank the management of Chiangrai Prachanukroh Hospital, Kamphaeng Phet Hospital, and Phayao Hospital for their support. We also thank Kevin Jones for his careful reading of the manuscript, critical review, and final check of the English language.

\section{SUPPLEMENTARY MATERIAL}

The Supplementary Material for this article can be found online at: https://www.frontiersin.org/articles/10.3389/fmed. 2021.719830/full\#supplementary-material

3. McHenry CR, Piotrowski JJ, Petrinic D, Malangoni MA Determinants of mortality for necrotizing soft-tissue infections. Ann Surg. (1995) 221:558-65. doi: 10.1097/00000658-19950500000013

4. Ozalay M, Ozkoc G, Akpinar S, Hersekli MA, Tandogan RN. Necrotizing soft-tissue infection of a limb: clinical presentation and factors related to mortality. Foot Ankle Int. (2006) 27:598-605. doi: 10.1177/1071100706027 00806 
5. Dworkin MS, Westercamp MD, Park L, McIntyre A. The epidemiology of necrotizing fasciitis including factors associated with death and amputation. Epidemiol Infect. (2009) 137:1609-14. doi: 10.1017/S0950268809002532

6. Anaya DA, McMahon K, Nathens AB, Sullivan SR, Foy H, Bulger E. Predictors of mortality and limb loss in necrotizing soft tissue infections. Arch Surg. (2005) 140:151-7. doi: 10.1001/archsurg.140.2.151

7. Roje Z, Roje Z, Matić D, Librenjak D, Dokuzović S, Varvodić J. Necrotizing fasciitis: literature review of contemporary strategies for diagnosing and management with three case reports: torso, abdominal wall, upper and lower limbs. World J Emerg Surg. (2011) 6:46. doi: 10.1186/1749-7922-6-46

8. Wong $\mathrm{CH}$, Yam $\mathrm{AK}$, Tan $\mathrm{AB}$, Song C. Approach to debridement in necrotizing fasciitis. Am J Surg. (2008) 196:e19-24. doi: 10.1016/j.amjsurg.2007.08.076

9. Hakkarainen TW, Kopari NM, Pham TN, Evans HL. Necrotizing soft tissue infections: review and current concepts in treatment, systems of care, and outcomes. Curr Probl Surg. (2014) 51:344-62. doi: 10.1067/j.cpsurg.2014.06.001

10. Khanna AK, Tiwary SK, Kumar P, Khanna R, Khanna A. A case series describing 118 patients with lower limb necrotizing fasciitis. Int J Low Extrem Wounds. (2009) 8:112-6. doi: 10.1177/1534734609334809

11. Hongladaromp C, Chareonsil B, Phadhana-anake O. Predictors on mortality from necrotizing fasciitis in Sawanpracharak Hospital, NakhonSawan, Thailand. Chiang Mai Med J. (2009) 48:135-142. Available online at: https:// he02.tci-thaijo.org/index.php/ThaiJSurg/article/view/241320 (accessed August 3, 2021).

12. Awsakulsutthi S. A retrospective review of necrotizing fasciitis in Thammasat University Hospital. J Med Assoc Thai. (2010) 93(Suppl 7): S246-53.

13. Giuliano A, Lewis F, Jr., Hadley K, Blaisdell FW. Bacteriology of necrotizing fasciitis. Am J Surg. (1977) 134:52-7. doi: 10.1016/0002-9610(77)90283-5

14. Bechar J, Sepehripour S, Hardwicke J, Filobbos G. Laboratory risk indicator for necrotising fasciitis (LRINEC) score for the assessment of early necrotising fasciitis: a systematic review of the literature. Ann R Coll Surg Engl. (2017) 99:341-6. doi: 10.1308/rcsann.2017.0053

15. El-Menyar A, Asim M, Mudali IN, Mekkodathil A, Latifi R, Al-Thani H. The laboratory risk indicator for necrotizing fasciitis (LRINEC) scoring: the diagnostic and potential prognostic role. Scand J Trauma Resusc Emerg Med. (2017) 25:28. doi: 10.1186/s13049-017-0359-Z

16. Su YC, Chen HW, Hong YC, Chen CT, Hsiao CT, Chen IC. Laboratory risk indicator for necrotizing fasciitis score and the outcomes. ANZ J Surg. (2008) 78:968-72. doi: 10.1111/j.1445-2197.2008.04713.x

17. Salvador VB, San Juan MD, Salisi JA, Consunji RJ. Clinical and microbiological spectrum of necrotizing fasciitis in surgical patients at a Philippine university medical centre. Asian J Surg. (2010) 33:51-8. doi: 10.1016/S1015-9584(10)60009-7

18. Wong CH, Khin LW, Heng KS, Tan KC, Low CO. The LRINEC (Laboratory Risk Indicator for Necrotizing Fasciitis) score: a tool for distinguishing necrotizing fasciitis from other soft tissue infections. Crit Care Med. (2004) 32:1535-41. doi: 10.1097/01.CCM.0000129486.35458.7D

19. Collins GS, Reitsma JB, Altman DG, Moons KGM. Transparent reporting of a multivariable prediction model for individual prognosis or diagnosis (TRIPOD): the TRIPOD Statement. BMC Med. (2015) 13:1. doi: $10.1186 / \mathrm{s} 12916-014-0241-\mathrm{z}$

20. Marcin JP, Romano PS. Size matters to a model's fit. Crit Care Med. (2007) 35:2212-3. doi: 10.1097/01.CCM.0000281522.70992.EF

21. Stevens DL, Bisno AL, Chambers HF, Dellinger EP, Goldstein EJ, Gorbach SL, et al. Practice guidelines for the diagnosis and management of skin and soft tissue infections: 2014 update by the Infectious Diseases Society of America. Clin Infect Dis. (2014) 59:e10-52. doi: 10.1093/cid/ciu444

22. Wang YS, Wong CH, Tay YK. Staging of necrotizing fasciitis based on the evolving cutaneous features. Int J Dermatol. (2007) 46:1036-41. doi: $10.1111 / j .1365-4632.2007 .03201 . x$

23. Haefeli M, Elfering A. Pain assessment. Eur Spine J. (2006) 15(Suppl. 1):S1724. doi: 10.1007/s00586-005-1044-x

24. Pain Management Guideline 2017. Best Practice Committee of the Health Care Association of New Jersey. Available online at: https://www.hcanj.org/ files/2013/09/Pain-Management-Guidelines-_HCANJ-May-12-final.pdf (accessed September 9, 2021).

25. Goyal S, Kanojiya R, Sharma M. Evaluation of risk factors for amputation and mortality in patients of necrotizing fasciitis. J Mahatma Gandhi
Univ Med Sci Technol. (2021) 5:38-46. doi: 10.5005/jp-journals-100570130

26. Nawijn F, Verhiel S, Lunn KN, Eberlin KR, Hietbrink F, Chen NC. Factors associated with mortality and amputation caused by necrotizing soft tissue infections of the upper extremity: a retrospective cohort study. World J Surg. (2020) 44:730-40. doi: 10.1007/s00268-019-05256-9

27. Khamnuan P, Chongruksut W, Jearwattanakanok K, Patumanond J, Tantraworasin A. Necrotizing fasciitis: epidemiology and clinical predictors for amputation. Int J Gen Med. (2015) 8:195-202. doi: 10.2147/IJGM.S82999

28. Moons KG, Harrell FE, Steyerberg EW. Should scoring rules be based on odds ratios or regression coefficients? J Clin Epidemiol. (2002) 55:1054-5. doi: 10.1016/S0895-4356(02)00453-5

29. Steyerberg EW, Vickers AJ, Cook NR, Gerds T, Gonen M, Obuchowski $\mathrm{N}$, et al. Assessing the performance of prediction models: a framework for traditional and novel measures. Epidemiology. (2010) 21:128-38. doi: 10.1097/EDE.0b013e3181c30fb2

30. Janssens AC, Deng Y, Borsboom GJ, Eijkemans MJ, Habbema JD, Steyerberg EW. A new logistic regression approach for the evaluation of diagnostic test results. Med Decis Making. (2005) 25:168-77. doi: 10.1177/0272989X05275154

31. Fagerland M, Hosmer D. A generalized hosmer-lemeshow goodness-of-fit test for multinomial logistic regression models. Stata J. (2012) 12:447-53. doi: $10.1177 / 1536867 X 1201200307$

32. Leisenring W, Pepe MS. Regression modelling of diagnostic likelihood ratios for the evaluation of medical diagnostic tests. Biometrics. (1998) 54:444-52. doi: $10.2307 / 3109754$

33. Abdullah M, McWilliams B, Khan SU. Reliability of the laboratory risk indicator in necrotising fasciitis (LRINEC) score. Surgeon. (2019) 17:309-18. doi: 10.1016/j.surge.2018.08.001

34. Leiblein M, Marzi I, Sander AL, Barker JH, Ebert F, Frank J. Necrotizing fasciitis: treatment concepts and clinical results. Eur J Trauma Emerg Surg. (2018) 44:279-90. doi: 10.1007/s00068-017-0792-8

35. Yaghoubian A, de Virgilio C, Dauphine C, Lewis RJ, Lin M. Use of admission serum lactate and sodium levels to predict mortality in necrotizing soft-tissue infections. Arch Surg. (2007) 142:840-6. doi: 10.1001/archsurg.142.9.840

36. Tan JH, Koh BT, Hong CC, Lim SH, Liang S, Chan GW, et al. A comparison of necrotising fasciitis in diabetics and non-diabetics: a review of 127 patients. Bone Joint J. (2016) 98-B:1563-8. doi: 10.1302/0301-620X.98B11.37526

37. Ahn J, Raspovic KM, Liu GT, Lavery LA, La Fontaine J, Nakonezny PA, et al. Lower extremity necrotizing fasciitis in diabetic and nondiabetic patients: mortality and amputation. Int J Low Extrem Wounds. (2019) 18:114-21. doi: $10.1177 / 1534734619836464$

38. Chang CP, Hsiao CT, Lin CN, Fann WC. Risk factors for mortality in the late amputation of necrotizing fasciitis: a retrospective study. World J Emerg Surg. (2018) 13:45. doi: 10.1186/s13017-018-0207-0

39. Herr M, Grabein B, Palm HG, Efinger K, Riesner HJ, Friemert B, et al. [Necrotizing fasciitis. 2011 update]. Unfallchirurg. (2011) 114:197-216. doi: 10.1007/s00113-010-1893-6

40. Mendelssohn DC, Barrett BJ, Brownscombe LM, Ethier J, Greenberg DE, Kanani SD, et al. Elevated levels of serum creatinine: recommendations for management and referral. CMAJ. (1999) 161:413-7.

Conflict of Interest: The authors declare that the research was conducted in the absence of any commercial or financial relationships that could be construed as a potential conflict of interest.

Publisher's Note: All claims expressed in this article are solely those of the authors and do not necessarily represent those of their affiliated organizations, or those of the publisher, the editors and the reviewers. Any product that may be evaluated in this article, or claim that may be made by its manufacturer, is not guaranteed or endorsed by the publisher.

Copyright $\odot 2021$ Chaomuang, Khamnuan, Chuayunan, Duangjai, Saokaew and Phisalprapa. This is an open-access article distributed under the terms of the Creative Commons Attribution License (CC BY). The use, distribution or reproduction in other forums is permitted, provided the original author(s) and the copyright owner(s) are credited and that the original publication in this journal is cited, in accordance with accepted academic practice. No use, distribution or reproduction is permitted which does not comply with these terms. 